Patients with tumors $>2 \mathrm{~cm}$ on the clinical examination had a 3.39-times higher hazard of death after relapse (HR, 3.39; 95\% CI, 1.52- 7.53) and the distant/both location of relapse had 2.23- times higher hazard of death (HR, 2.23; 95\% CI, 1.14- 4.36)

The 2-years survival rates after relapse were $76 \%$ for tumors $<2 \mathrm{~cm}, 50.0 \%$ tumors $>2 \mathrm{~cm}$ on the clinical examination, $76 \%$ for local relapse and $47 \%$ for distant/both location relapse.

Conclusion* The tumor size on clinical examination, the location of relapse, the histologic subtype and the treatment with Bevacizumab, modify the risks of death after relapse on patients with cervical cancer IB1. Tumor $>2 \mathrm{~cm}$ on clinical examination and distant recurrences have a shorter survival time after relapse

\section{TRANSITION FROM FIGO-2009 TO FIGO-2018 IN WOMEN WITH EARLY-STAGE CERVICAL CANCER; DOES THE REVISED STAGING CORRECTLY REFLECT RISK GROUPS?}

\begin{abstract}
${ }^{1 ; 2 ; 3}$ S Sponholtz*, 4;50 Mogensen, ${ }^{1 ; 6 ; 7} \mathrm{M}$ Hildebrandt, ${ }^{1 ; 8} \mathrm{D}$ Schledermann, ${ }^{9} \mathrm{E}$ Parner, ${ }^{2} \mathrm{~A}$ Markauskas, ${ }^{10} \mathrm{~L}$ Froeding, ${ }^{4} \mathrm{~K}$ Fuglsang, ${ }^{6} \mathrm{~J} \mathrm{Holm},{ }^{4 ; 5} \mathrm{~S}$ Bjørnholt, ${ }^{1 ; 4 ; 5} \mathrm{P}$ Jensen. ${ }^{1}$ University of Southern Denmark, Institute of Clinical Research, Odense C, Denmark; ${ }^{2}$ Odense University Hospital, Department of Gynaecology and Obstetrics, Odense C, Denmark; ${ }^{3}$ Odense University Hospital and Region of Southern Denmark, Open Patient Data Explorative Network, Odense C, Denmark; ${ }^{4}$ Aarhus University Hospital, Department of Gynaecology and Obstetrics, Aarhus N, Denmark; ${ }^{5}$ Aarhus University, Institute of Clinical Medicine, Aarhus N, Denmark; ${ }^{6}$ Odense University Hospital, Department of Nuclear Medicine, Odense C, Denmark; ${ }^{7}$ Odense University Hospital and University of Southern Denmark, Centre for Innovative Medical Technology, Odense C, Denmark; ${ }^{8}$ Odense University Hospital, Department of Pathology, Odense C, Denmark; ${ }^{9}$ Aarhus University, Department of Public Health, Aarhus C, Denmark; ${ }^{10}$ Copenhagen University Hospital, Department of Gynaecology, Kbh Ø, Denmark
\end{abstract}

\subsection{6/ijgc-2021-ESG0.47}

Introduction/Background* The International Federation of Gynaecology and Obstetrics (FIGO) revised cervical cancer staging in 2018. We aimed to evaluate risk factors associated with lymph node macro- and micrometastases in women with early-stage cervical cancer, focusing on the revised FIGO-2018 staging system. The overall purpose was to evaluate if the stage migration related to the implementation of FIGO-2018 correctly reflects risk groups as indicated by the presence of lymph node metastases.

Methodology Using data from a national prospective cohort study on sentinel lymph node (SLN) mapping in 245 women with early-stage cervical cancer, we reallocated women from FIGO-2009 to FIGO-2018 stages. We used binary and multiple regression models to investigate the risk ratio of FIGO2018 stages and tumour characteristics associated with nodal metastases.

Result(s)* Stage migration occurred in 80.4\% (197/245), due to tumour size or depth of invasion in $75.1 \%(148 / 197)$, nodal metastases in $19.3 \%$ (38/197), and imaging in $4.5 \%$ $(11 / 245)$. Downstaging to FIGO-2018 IA stages occurred in $36,7 \%$ (90/245). Six (5.7\%) women with stage IA tumour characteristics were upstaged to IIIC1 due to the findings of nodal metastases. The depth of invasion ranged from $4-5 \mathrm{~mm}$ and the tumour size from $9-22 \mathrm{~mm}$; all six metastases were SLNs. For the whole population, risk factors significantly associated with nodal metastases were FIGO-2018 $\geq$ IB2 $(\mathrm{p}<$
$0.001)$, parametrial invasion $(\mathrm{p}<0.001)$, and lymphovascular space invasion (LVSI) $(\mathrm{p}<0.001)$. All three remained significantly associated with nodal metastases in a multivariate analysis.

Conclusion* The FIGO-2018 revised staging system causes stage migration for a large proportion of women with earlystage cervical cancer. The attention on depth of invasion rather than horizontal dimension seems to reflect the risk of nodal metastases correctly. The use of sentinel node mapping in stage IA FIGO-2018 appears to be justified.

\section{ROUTINE USE OF CYTOKERATIN IMMUNOHISTOCHEMISTRY IMPROVES THE DETECTION OF LOW VOLUME DISEASE IN EARLY-STAGE CERVICAL CANCER BUT IS COSTLY}

${ }^{1}$ I Baeten*, ${ }^{1} \mathrm{~J}$ Hoogendam, ${ }^{2} \mathrm{G}$ Jonges, ${ }^{3} \mathrm{~A}$ Braat, ${ }^{2} \mathrm{P}$ Van Diest, ${ }^{1} \mathrm{~K}$ Gerestein, ${ }^{1} \mathrm{R}$ Zweemer. ${ }^{1}$ University Medical Center Utrecht, Department of Gynaecological Oncology, Utrecht, Netherlands; '2University Medical Center Utrecht, Department of Pathology, Utrecht, Netherlands; ${ }^{3}$ University Medical Center Utrecht, Department of Radiology and Nuclear Medicine, Utrecht, Netherlands

\subsection{6/ijgc-2021-ESG0.48}

Introduction/Background* In cervical cancer, the sentinel lymph nodes (SLNs) are processed according to the pathological ultrastaging protocol. As part of this protocol immunohistochemistry with cytokeratin AE1/AE3 is performed in addition to standard hematoxylin and eosin (H\&E) staining, aiding the detection of low volume disease (i.e. micrometastases and isolated tumour cells (ITC)). Current guidelines advise routine use of cytokeratin immunohistochemistry. We studied the pathological yield, in terms of detecting low volume disease, and cost-effectiveness of this routine immunohistochemistry use.

Methodology We retrospectively included all FIGO stage IAIIA1 cervical cancer patients who had undergone SLN procedures at our institution between 2007 and 2020. Pathological data were collected from every patient including the number of SLNs stained with cytokeratin imunohistochemistry. Data were analysed using descriptive statistics and McNemar test.

Result(s)* In total 232 cervical cancer patients had undergone a successful SLN procedure harvesting a total of 647 SLNs. Of these nodes, 540 SLNs from 215 patients were routinely processed with cytokeratin immunohistochemistry. Immunohistochemistry identified low volume disease in 25 SLNs from 22 patients: 14 with micrometastases (11 patients) and 11 with ITC (11 patients). Four nodes with micrometastases (three patients) and six nodes with ITC (six patients) would have been missed without the routine use of immunohistochemistry. Overall, 54 SLNs needed to be immunohistochemically stained to detect one additional SLN with low volume disease, 135 for micrometastases and 90 for ITC, leading to an expenditure of $€ 5920$ to identify one additional low volume diseased SLN: $€ 14800$ for micrometastases and $€ 9867$ for ITC. Compared to H\&E staining, routine immunohistochemistry significantly increased the rate of patients with low volume disease from $18(8.4 \%)$ to 26 patients $(12.1 \%)$ $(p=0.02)$. When only micrometastases were considered as tumour positive, routine immunohistochemistry increased the rate of patients with positive sentinel lymph nodes from 12 $(5.6 \%)$ to 15 patients $(7.0 \%)(p=0.25)$. 